\title{
DIAPHRAGMATIC and CONTROLLED BREATHING
}

\author{
C. P. RODSETH*
}

*M.Sc. (Strathclyde), B.Sc. (Physio.) (Wits.), M.C.S.P., Dip. T.P. Senior Lecturer in Physiotherapy, University of Cape Town.

(This paper has been edited for the purposes of publication).

By this title I understand that I am to discuss the general field of what is also called localised breathing.

What is meant by localised breathing?

I shall assume, to begin with, that localised breathing means that by voluntarily moving areas of the walls of the thoracic cavity the underlying lung tissue is selectively aerated.

This assumption is based at least on implications if not frank statements to this effect by the early writers on chest physiotherapy. For example, in the early Brompton Hospital pamphlets on exercises for asthma and bronchitis" they say, apropos of "expansion of the lower chest"... "This exercise will help the patient to make better use of the bases of the lungs. ." or in their booklet "upper lateral expansion" is said to be "for expansion of lower part of upper lobe".

Winifred Thacker in the 3rd (1971) edition of her book on postural drainage and respiratory control ${ }^{3}$ says, under the heading "mechanism of respiration": "As the thorax becomes enlarged by the action of the muscles of inspiration, so the lungs expand to follow the movements of the walls"; then later: "From this explanation, it is obvious that localised breathing is dependent on controlled rib movements . . ." On a simple, unsophisticated basis these seemed quite reasonable assumptions to make and gave us a comforting mechanical rationale for our treatments.

However, it has not been possible to demonstrate this effect experimentally and even on theoretical grounds; in the healthy chest it would not seem to be possible.

As the thorax increases in volume, so does the underlying lung, but since sliding is easily possible between the two layers of pleura, the distribution of air is governed by factors within the lung and not by the position of a localised increase in volume. In other words, an increase in volume will be a general increase and the lung will accommodate generally. Should there be some volume of tissue within the lung which is "stiffer" than its surroundings, then the surrounding lung will expand slightly more to compensate.

It should be borne in mind, however, that there is a fundamental variation in local aeration due to gravity, In the erect position, at the end of expiration with the glottis open, i.e. with the lungs in the state of Functional Residual Volume (F.R.C.), the weight of the lung, supported only at the hilum, tends to pull away from the apical surface and fall towards the diaphragmatic surface. Thus, at F.R.C., the individual alveoli in the apical region have a greater volume than those basally. However, the effect of gravity on the circulation is such as to cause it to tend to pool towards the bases of the lungs in the erect posture, so increasing the perfusion rate (uptake of $\mathrm{O}_{2}$ and release of $\mathrm{CO}_{2}$ ) in this area.

Failure to detect voluntary local aeration has been reported by Grimby et al. ${ }^{5}$ They summarise by saying that "although diaphragmatic and basal costal breathing

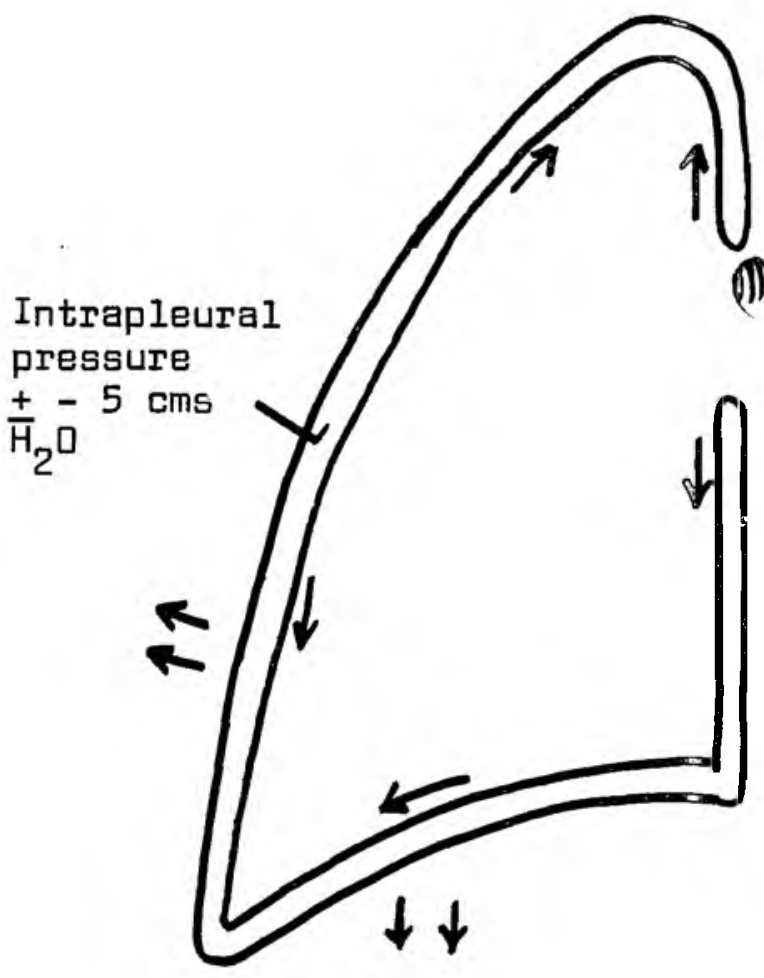

Diagram I

involves a change in the chest wall shape, no systematic effects on ventilation distribution have yet been demonstrated"' In 1975 two fourth year physiotherapy students at the University of Cape Town, Groves and Larn, using radio-active Xenon gas and localised lower lateral costal breathing on six trained normal subjects failed to show any alteration in normal distribution of ventilation during localised breathing. This situation is also recognised by Gaskell ${ }^{7}$ who says apropos breathing control: "physiotherapists must be prepared to change the rationale of their techniques as more experiments are performed in this field".

It can be said, however, that on theoretical grounds if pleural adhesions are present, preventing much sliding between the pleural layers, then localised movement of the chest wall may well be able selectively to expand underlying, physically attached volumes of lung. I am told by pathologists that, at post-mortem, a large percentage of people (especially the elderly) do have pleural 


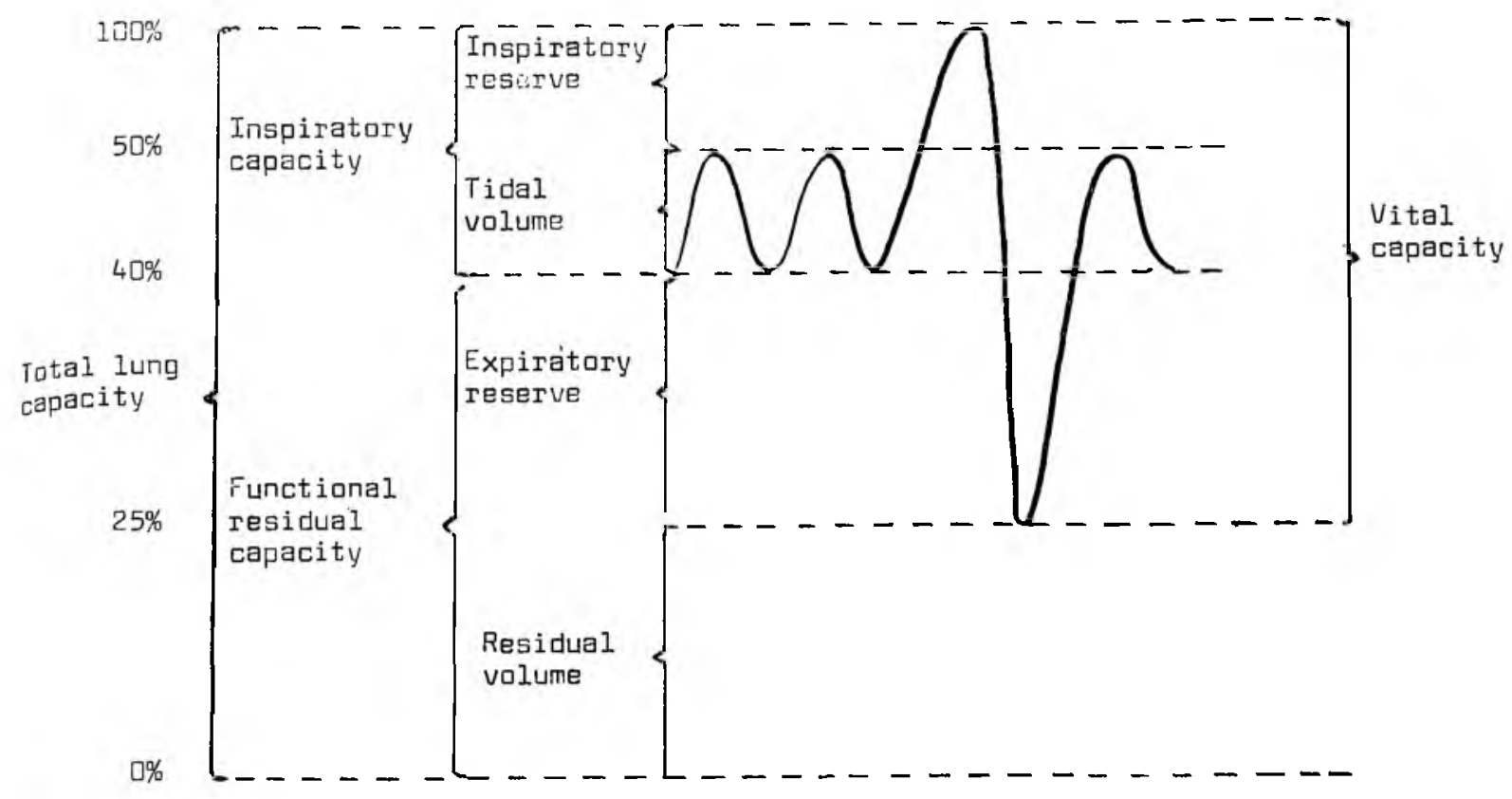

Diagram 2: Lung valumes

adhesions; even more especially if they have had some peripheral infective process in the lung. I emphasise that I know of no experimental work to confirm this idea but it does seem to be a possibility.

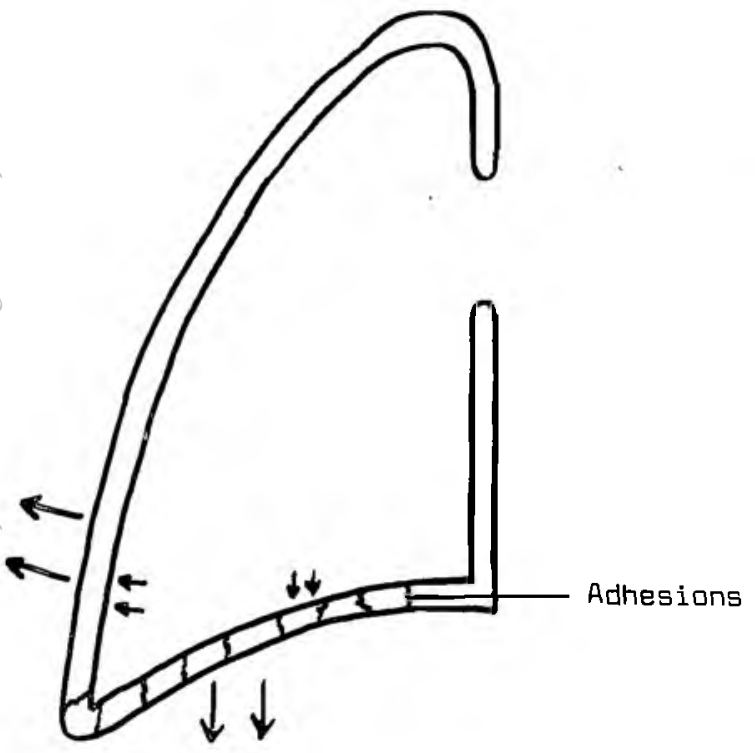

Diagram 3
What, then, of disphragmatic and controlled breathing? There is no doubt as to the clinical value of breathing exercises; irrespective of the results of lung function tests the patient's day-to-day functional ability improves.

Breathing exercises are beneficial in:

1. Aiding relaxation and relief of anxiety.

2. Slowing the respiratory rate which improves bloodgas exchange without significant increase in energy cost.

3. Eliminating purposeless unco-ordinated associated respiratory movements which use unnecessary energy.

4. Controlling breathing and improving exercise tolerance.

Breathing is a co-ordinated muscular activity whose purpose is to move volumes of gas into and out of the lungs and in so doing to use up as little of the inspired oxygen as possible so that the remainder is available for other bodily activities.

Thus, when we are presented with patients whose respiratory mechanism has been damaged in such a way as to increase the effort required to breathe, not only is there a mechanical problem to deal with but often also one of the control of breathing. This action, normally automatic, now comes under constant voluntary control by the patient, resulting frequently in poor coordination and increased oxygen consumption due to apprehension, bad posture and lack of understanding of the underlying mechanisms. Some emphysematous patients will discover the benefits of "pursed-lips" breathing for themselves but the majority of patients with chronic lung disease, in my experience, will aggravate their condition with bad mannerisms unless corrected.

I suggest, therefore, that we teach diaphragmatic and controlled breathing not because we wish the patient to expand the bases or any other region of his lungs selectively, but because the use of the diaphragm en- 
ables him to move the largest volumes of air most efficiently (as normal subjects do in quiet, subconscious breathing) and because control of this will eliminate unnecessary muscle work by accessory muscles of respiration, resulting in a more functional patient.

With regard to function, I would also draw to your attention the work on exercise physiology reflected in the "Trailing Coat-Tails" column of the South African Medical Journal of 17th January, 1976, in which Noakes draws attention to the ability of muscle cells themselves to adapt by being "trained" to require less oxygen for contraction. This adaptation takes the form of an increase in skeletal muscle mitachondria. This new emphasis on the importance of peripheral adaptation as opposed to central cardio-respiratory-vascular adaptations to increased work load provides a possible explanation for the success of physiotherapy breathing exercises and for the increasing "exercise tolerance" which is clinicaly demonstrated in the face of unchanged pulmonary function tests. The changes are taking place in the skeletal muscles and not in the heart or lungs!

\section{Conclusion}

I would conclude by making the point that I have attempted to draw attention to the shift in reasoning for the efficacy of diaphragmatic and controlled breathing without calling into question the efficacy of such activities. The emphasis is now on economy of movement. This is based on experiments on normal lungs and it is freely admitted that a great deal of quantitative investigation still remains to be done on damaged lungs. However, it has been shown that very little basis exists for claiming selective expansion of segments or even lobes of lungs (especially healthy ones) and so I would suggest that treatment time be devoted more to general breathing re-education, using primarily the diaphragm, and that over-particular localised breathing be deleted. The aim is to train a pattern of breathing movement. It also seems that the general activity exercises given to patients with chronic destructive and obstructive lung disease can benefit them functionally by training their muscles to manage on the reduced amount of oxygen with which the lungs can supply them.

I have not discussed postural drainage and the localised breathing which often accompanies it. I am sure this is a topic which would also prove most interesting to elucidate.

\section{References}

1. Brompton Hospital, Physiotherapy Department, Children's Exercises for Asthma and Bronchitis. Undated, received by author in 1967.

2. Gaskell, D. V. (1964), Brompton Hospital, Physiotherapy for Medical and Surgical Thoracic Conditions, Second Revision, p. 5.

3. Thacker, W. (1971), Postural Drainage and Respiratory Control, 3rd ed., London, Lloyd-Luke.

4. Cherniak, R. M., Chernia. L., Naimarf, A. (1972), Respiration in Health and Disease, 2nd ed., Philadelphia, W. B. Saunders.

5. Grimby, G., Bake, Dempsy, J. (1974), Aspects of Lung Expansion in Relation to Pulmonary Physiotherapy, Amer. Rev. Resp. Chest Dis., Dec., No. 2, Part 2, pp. 145-153.

6. Groves, L., Larn, A. (1975), A Quantitative Evaluation of the Distribution of Regional Ventilation During Localised Breathing, B.Sc. (Phys.), Thesis, U.C.T., Sept., 1975.

7. Gaskell, D. V. (1975), Introduction to the Treatment of Medical Chest Conditions, Chap. 5 in Chest, Heart and Vascular Disorders for Physiotherapists ed. J. E. Cash, London, Faber and Faber.

8. Noakes, T. D. (1976), Exercise Physiology, S. Afr. Med. J., 50, 59.

\section{COUGHING}

\section{J. RUNNALLS*}

*Dip. Physiother., C.T.P. (Cape Town), Senior Lecturer of Stellenbosch and Tygerberg Hospital.

Physiotherapists who have worked in surgical wards are surely au fait with the most frustrating fact, that invariably, after spending some time with a patient, this particular patient coughs once she has moved on to treat the patient in the next bed! In fact this has almost tended to become my standard practice: if 1 persevere and demand gentle diaphragmatic breathing over a long enough period, the patient eventually coughs spontaneously. Similarly, physiotherapists involved in the treatment of patients suffering from diffuse obstructive airways syndrome will have noticed that these patients cough and cough, but oh so ineffectively.

The fact that passive movements and movement provoke coughing in the unconscious patient is also a fait accompli. These simple observations seem clearly to indicate that the physiotherapist should possibly think further before saying "breath in deeply, now cough". There is evidence that we could be far more specific when we demand coughing of our patients, specific in our teaching of the technique of coughing per se; specific with regard to treatment techniques; and specific in what we demand. Coughing is not always an easy or a pleasant procedure for the patient, and should therefore be as directed, and as non-drastic a procedure as possible. Schamroth (1976), "The cough is a complex, highly co-ordinated act that results in the very rapid expulsion of alveolar gas at a very high velocity, presumably to sweep the airway free of irritant gases, dust, smoke, excess mucus, cell débris or pus. The first step is similar to the Valsalva manoeuvre - by a forced expiratory effort against a closed glottis, the patient develops high intrathoracic and intrapulmonic pressure. The glottis then opens abruptly so that there is a large pressure difference between alveolar pressure and upper tracheal pressure (now atmospheric). This results in a rapid flow rate. Equally important is dynamic compression of intrathoracic airways, including the trachea, due to high intrathoracic pressure $\left(+40 \mathrm{~mm} \mathrm{H}_{2} \mathrm{O}\right)$." Comrog (1974):" Furthermore, Comroe, also states that during coughing the non-cartilaginous part of the trachea is inverted by the forces of dynamic compression. This increases the velocity of the air passing through so that the linear velocity actually increases from $667 \mathrm{~cm} / \mathrm{sec}$. to $28000 \mathrm{~cm} / \mathrm{sec}$. (500 miles per hour or $85 \%$ the speed of sound!) This sudden rush of air is responsible for the dislodging of foreign material.

Brink and De Kock (1973), state that "The nerve ends of the upper airways are sensitive to contact with foreign matter, changes in volume and noxious gases. As the acinus has no nerve supply, pathology of the acinus will only stimulate coughing once the material reaches the upper airways. The larynx, carina and bifurcation of the larger bronchi are the most sensitive parts of the airways". Extremely limiting deformities of the trachea in the presence of diffuse obstructive airways syndrome have also been demonstrated by De Kock (1976)."

Leith (1968), ${ }^{5}$ has several interesting suggestions. He maintains "The object of the game in coughing is to pump a 'liquid' up through a tube, using energy transformed from a gas, also moving up through a tube". 